\title{
Evaluación in vitro de la resistencia compresiva de un sellante resinoso fluorado pre y post liberación de flúor
}

\author{
In vitro compressive strength of fluoride-containing resin-based sealant before and \\ after fluoride release
}

Vergara $\mathrm{C}^{1}$, Uribe $\mathrm{S}^{2}$

\begin{abstract}
RESUMEN
Objetivo: Evaluar in vitro la resistencia compresiva de un sellante resinoso fluorado ( $F)$ pre y post liberación de flúor luego de la inmersión en agua. Método: Se utilizaron 40 probetas de $6 \mathrm{~mm}$ de alto y $20 \mathrm{~mm}$ de diámetro de sellante resinoso fluorado (FluroShield, Brasil, Dentsply) y de sellante no fluorado (Concise(TM) Light Cured White Sealant, USA, 3M ESPE). Las probetas fueron divididas en cuatro grupos, dos de sellante resinoso fluorado (F1 y F2) y dos de control (C1 y C2). Un grupo de probetas de sellante fluorado y un grupo del control (F1/C1) se mantuvieron sin exposición al agua, mientras que los grupos restantes (F2/C2) fueron inmersos en agua destilada por 30 días. Se midió la liberación de flúor desde el grupo F2 mediante el método de electrodo selectivo los días 1, 2, 3 y 30. Posteriormente se midió la resistencia compresiva mediante una máquina de ensayos mecánicos universales (Lloyd, LR 100, UK) con una velocidad de cruceta de $1 \mathrm{~mm} / \mathrm{min}$. Las comparaciones entre los grupos F1 vs F2 y C1 vs C2 se analizaron con t-Student. El nivel de significancia se estableció a 0.05. Resultados: La resistencia compresiva en MPa antes y después de la inmersión en agua para el sellante resinoso fluorado fue 337.2 y 337.4 , mientras que la del sellante control fue 203.8 y 213.4 . Para ambos grupos las diferencias observadas no fueron significativas. Se observó un patrón de liberación de flúor inicial de $1.9 \mathrm{ppm}$ durante las primeras 24 horas para luego decaer a $0.0 \mathrm{ppm}$ al tercer día de liberación. Conclusión: La liberación de flúor desde un sellante resinoso fluorado no afectó su resistencia compresiva en este estudio in vitro.
\end{abstract}

Rev. Clin. Periodoncia Implantol. Rehabil. Oral Vol. 5(1); 9-12, 2012.

Palabras clave: Fluoruros, sellantes de fosas y fisuras, resistencia compresiva, in vitro.

\section{ABSTRACT}

Aim: To evaluate in vitro compressive strength of fluoride-containing resin-based sealant $(F)$ before and after fluoride release in water. Materials and Methods: We used 40 specimens with $6 \mathrm{~mm}$ of height and $20 \mathrm{~mm}$ of diameter using fluoride-containing resin-based sealant (FluroShield,Brasil, Dentsply) and non-fluoride-containing resin-based sealant (Concise (TM) Light Cured White Sealant, USA, 3M ESPE). The specimens were divided into four groups, two fluoride-containing resin-based sealants (F1 and F2) and two like control groups (C1 and C2). A group of specimens of fluoride-containing resin-based sealant and a control group (F1/C1) remained without exposure to water, while other groups (F2/C2) were immersed in distilled water for 30 days. The release of fluoride from the F2 was measured through selective electrode method on days 1, 2, 3 and 30. Subsequently, the compressive strength was measured using a universal testing machine (Lloyd, LR 100, UK) with a crosshead speed of $1 \mathrm{~mm} / \mathrm{min}$. Comparisons between groups F1 vs F2 and C1 vs C2 were analyzed with t-Student. The significance level was set at 0.05 . Results: The compressive strength (Mpa) before and after immersion in water for fluoride-containing resin-based sealant was 337.2 and 337.4 , while the control sealant was 203.8 and 213.4. For both groups the observed differences were not significant. The initial fluoride release was $1.9 \mathrm{ppm}$ during the first 24 hours and then declined to $0.0 \mathrm{ppm}$ during the third day of release. Conclusion: In this in vitro study, the release of fluoride from a fluoride-containing resin-based sealant does not affect compressive strength.

Rev. Clin. Periodoncia Implantol. Rehabil. Oral Vol. 5(1); 9-12, 2012.

Key words: Fluorides, pit and fissure sealants, compressive strength, in vitro.

\section{INTRODUCCIÓN}

Entre el $67 \%$ al $90 \%$ de todas las lesiones de caries en niños y adolescentes, se desarrollan en la superficie oclusal de molares ${ }^{(1)}$. Para prevenir estas lesiones cariosas se utilizan sellantes de fosas y fisuras, los que en recientes revisiones sistemáticas, han mostrado cerca de un $80 \%$ de reducción de caries oclusales ${ }^{(2)}$ e incluso en la detención de lesiones oclusales no cavitadas ${ }^{(3)}$.

El éxito del sellante se basa en la retención e integridad de este material en las irregularidades producidas por el grabado ácido en la superficie del esmalte ${ }^{(4,5)}$, por lo que requiere propiedades mecánicas que le permitan soportar las fuerzas compresivas que ocurren durante el proceso masticatorio ${ }^{(6)}$. Si un paciente pierde, total o parcialmente, un sellante, ocurrirá el contacto entre la superficie del esmalte y los ácidos provenientes del metabolismo del biofilm, iniciándose el proceso de desmineralización del esmalte ${ }^{(7)}$. Desde la década del 70 , se ha estudiado la incorporación de fluoruro de sodio $(\mathrm{NaF})$ al sellante resinoso, con la finalidad de que la disolución de esta sal proporcione iones fluoruros que brinden una protección adicional en los casos que se pierda la unión resina-esmalte ${ }^{(8)}$. Por esto, la finalidad de incorporar fluoruros a un sellante de resina sería aumentar la eficacia preventiva del sellante. Este efecto sería logrado cuando el sellante resinoso fluorado liberase el flúor al ser inmerso en un ambiente húmedo. Sin embargo, un reporte previo muestra que la liberación de flúor en composites, cementos de ionómero vítreo y compómeros está correlacionado negativamente con las propiedades mecánicas de estos biomateriales ${ }^{(9)}$.

Por lo tanto, se evaluará in vitro el efecto de la liberación de flúor en la resistencia compresiva de un sellante resinoso fluorado luego de ser inmerso en un ambiente acuoso.

1. Alumna Odontología. Escuela de Odontología. Alumna Programa de Honor en Investigación. Facultad de Medicina, Universidad Austral de Chile. Valdivia, Chile.

2. Profesor Asociado. Escuela de Odontología, Facultad de Medicina, Universidad Austral de Chile. Valdivia, Chile.

Correspondencia autor: Sergio Uribe Espinoza. sergiouribe@uach.cl. Cátedra de Cariología, Escuela de Odontología, Facultad de Medicina, Universidad Austral de Chile. Valdivia, Chile. Rudloff \#1640, Valdivia, Chile. Trabajo recibido el 24/10/2011. Aprobado para su publicación el 15/03/2012. 


\section{MATERIAL Y MÉTODO}

Preparación de las Muestras

Para realizar esta investigación se eligió un sellante resinoso fluorado (F) y otro no fluorado utilizado como grupo control (C). Los materiales seleccionados debían ser fotopolimerizables, de color blanco y en el caso de F, que tuviese como sal fluorada fluoruro de sodio (NaF) debido a que ésta es la sal fluorada que cuenta con más estudios en su incorporación-liberación desde el sellante. Se utilizó un sellante fluorado, cuya concentración de flúor liberada es conocida(10), y no ha sido evaluado previamente su resistencia compresiva ${ }^{(6)}$. Además de lo anteriormente señalado, de acuerdo a la disponibilidad de materiales encontrada en la ciudad de Valdivia en marzo de 2011, se incluyeron los siguientes materiales indicados en la Tabla 1. de sellante resinoso y un grupo del control (F1/C1) se mantuvieron sin exposición al agua, mientras que los grupos restantes (F2/C2) fueron inmersos en agua destilada por 30 días. Se midió la liberación de flúor desde el grupo F2 mediante el método de electrodo selectivo los días 1, 2, 3 y 30 . Posteriormente se midió la resistencia compresiva (ver Figura 1).

\section{Almacenamiento}

Las probetas fueron almacenadas en una caja hermética, de color negro, a temperatura ambiente $\left(20^{\circ} \mathrm{C}\right)$. Dos grupos $(\mathrm{F} 1$ y C1, $\mathrm{n}=20$ ) fueron almacenados por 48 horas y posteriormente sometidos al test de resistencia compresiva según se describe más adelante. Los grupos restantes ( $F 2$ y C2, $n=20$ ) fueron almacenados por 48 horas y posteriormente sometidos al proceso de liberación de flúor.

Tabla 1. Composición de los sellantes resinosos utilizados en este estudio.

\begin{tabular}{|l|l|l|l|l|l|l|}
\hline Nombre & Tipo & Composición & $\begin{array}{l}\text { Porcentaje } \\
\text { de Relleno }\end{array}$ & Fabricante & Lote & $\begin{array}{l}\text { Fecha de } \\
\text { Vencimiento }\end{array}$ \\
\hline $\begin{array}{l}\text { FluroShield, } \\
\text { matizado. }\end{array}$ & Fluorado (F) & $\begin{array}{l}\text { Urethane-modified } \\
\text { Bis-GMA, } \\
\text { triethylene glycol } \\
\text { dimethacrylate, } \\
\text { aluminum and } \\
\text { barium borosilicate, } \\
\text { phosphoric acid } \\
\text { tetracyclic ester, } \\
\text { sodium fluoride, } \\
\text { N-methyl } \\
\text { dietanolamine, } \\
\text { camphoroquinone }\end{array}$ & $\begin{array}{l}\text { Dentsply } \\
\text { (Petrópolis, } \\
\text { RJ, Brazil) }\end{array}$ & 263503 C & Julio, 2011 \\
\hline $\begin{array}{l}\text { Concise(TM) } \\
\text { Light Cured } \\
\text { White } \\
\text { Sealant }\end{array}$ & $\begin{array}{l}\text { No fluorado } \\
\text { (C) }\end{array}$ & $\begin{array}{l}\text { Triethylene glycol } \\
\text { dimethacrylate, } \\
\text { Bisphenol a } \\
\text { diglycidyl ether } \\
\text { dimethacrylate } \\
\text { (bisgma), Silane } \\
\text { treated silica. }\end{array}$ & $5-10 \%$ & $\begin{array}{l}3 \mathrm{M} \text { ESPE } \\
\text { (St. Paul, } \\
\text { MN, USA) }\end{array}$ & $\begin{array}{l}\text { N167 } \\
244 .\end{array}$ & Mayo, 2013. \\
\hline
\end{tabular}

Se utilizaron 20 jeringas de $F$ y 6 botellas de $C$ con las que un operador (CV) preparó 44 probetas utilizando un molde metálico cilíndrico que permitió la confección de cuerpos de prueba cilíndricos de $6 \mathrm{~mm}$ de alto y $20 \mathrm{~mm}$ de diámetro sobre una loseta de vidrio. Se aplicó vaselina líquida tanto en el interior del molde como en la superficie de la loseta. Cada incremento de $2 \mathrm{~mm}$ fue fotopolimerizado por 20 segundos utilizando una lámpara halógena (QHL 75, Dentsply, Alemania). Posteriormente, el molde cilíndrico junto al contenido de sellante fueron alejados de la loseta. Las probetas fueron retiradas presionando desde una cara de la probeta en el interior del molde metálico con un objeto romo hasta la salida completa de la probeta desde el interior del molde.

Las probetas fueron divididas en cuatro grupos, dos de sellante resinoso fluorado (F1 y F2) y dos de control (C1 y C2). Un grupo de probetas

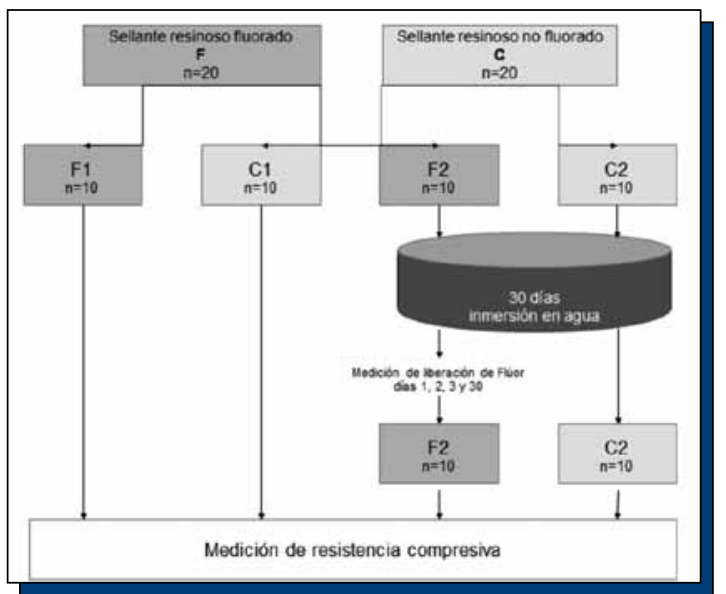

Figura 1. Esquema que indica como las probetas de los sellantes fueron agrupadas para el sellante resinoso fluorado $(F)$ y no fluorado $(C)$. Los grupos $F 1$ y $C 1$ fueron sometidos a la prueba de resistencia compresiva sin ser inmersos en agua. Los grupos F2 y C2 fueron inmersos en agua por 30 días y posteriormente fueron sometidos a la prueba de resistencia compresiva. Debido a que el grupo F2 correspondía al grupo de sellante liberador de flúor, se midieron las concentraciones de flúor liberadas en el medio de inmersión (agua destilada) los días 1, 2, 3 y 30.

\section{Liberación de Flúor ${ }^{(6)}$}

La unidad de medida de los datos obtenidos para la concentración de flúor liberado fue partes por millón (ppm). Se utilizó el método de electrodo selectivo de iones de flúor utilizando un electrodo (9609 BNC, Orion, Thermo Scientific, USA) conectado a un analizador de iones (Orion 5 Star, Orion, Thermo Scientific, Singapore) y a una sonda de temperatura (Orion 927005MD Epoxy ATC Probe, Orion, Thermo Scientific, USA). El electrodo de flúor fue mantenido de acuerdo a las instrucciones del fabricante y calibrado antes de realizar cada medición, utilizando soluciones estándar preparadas diariamente por un operador (CV) a concentraciones de $0.25 \mathrm{ppm}, 0.5 \mathrm{ppm}, 1$ $\mathrm{ppm}$ and $2 \mathrm{ppm}$. Las soluciones fueron obtenidas utilizando una solución stock de F a 202.7 ppm (Merck 13-01-10).

Cada probeta del grupo F2 $(n=10)$ fue inmersa en 30 $\mathrm{ml}$ de agua destilada en un vaso precipitado por 24 horas a temperatura ambiente $\left(20^{\circ} \mathrm{C}\right)$. Posteriormente cada vaso precipitado fue cubierto con película de laboratorio (Parafilm M) y almacenado en oscuridad. Luego de 24 horas cada probeta fue tomada con una pinza plástica y enjuagada con $20 \mathrm{ml}$ de agua destilada en el vaso original colectando finalmente $50 \mathrm{ml}$. Los volúmenes de agua utilizados fueron medidos mediante 3 micropipetas (Eppendorf research.

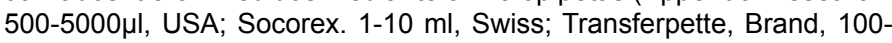
$1000 \mu l$, Germany). Después de esto, cada probeta fue inmersa en un nuevo vaso con $30 \mathrm{ml}$ de agua destilada. Todas las soluciones fueron preparadas a temperatura estándar $\left(20^{\circ} \mathrm{C}\right)$ utilizando un circulador de inmersión (Model 112A, VWR, USA).

La medición de concentración de flúor liberado del grupo F2 se realizó utilizando el electrodo selectivo de ión flúor. Para esto, se añadió $5 \mathrm{ml}$ de solución buffer de ajuste iónico (TISAB III 940911, Orion lon plus, Thermo Scientific, USA) a cada vaso precipitado $(n=10)$ que ya contenía el agua fluorada liberada por cada probeta. Para lograr homogeneizar el contenido de flúor dentro de la solución y a la vez mezclar la solución de cada muestra con la solución buffer de forma estandarizada, se utilizó agitación magnética. La agitación magnética consistió en ubicar una barra magnética a cada vaso precipitado $(n=10)$ y ubicar cada vaso sobre una máquina agitadora (LMS-1001, LabTech, Daihan Lab. Tech, Korea) por 5 segundos a velocidad media. Después, se retiró la barra magnética con una pinza plástica y se posicionó el electrodo en el contenido líquido, con la precaución de evitar la formación de burbujas en la superficie del electrodo. Posteriormente, se realizó la lectura de cada muestra 3 veces. La membrana del electrodo fue enjuagada con agua destilada y secada con papel absorbente entre las mediciones. Las mediciones de flúor se realizaron los días 1, 2, 3 y 30 . Durante el periodo comprendido entre el día 3 y 30 las muestras se mantuvieron sin recambio de solución y con agitación manual una vez al día.

\section{Grupo Control}

C1 fue sometido exactamente a las mismas condiciones que $\mathrm{F} 1$. En el caso del grupo $\mathrm{C} 2$ fue inmerso en $30 \mathrm{ml}$ de agua destilada en un vaso precipitado por 24 horas a temperatura ambiente $\left(20^{\circ} \mathrm{C}\right)$. Posteriormente cada vaso precipitado fue cubierto con película de laboratorio (Parafilm M) y almacenado en un recinto oscuro. Luego de 24 horas cada probeta fue tomada con una pinza plástica e inmersa en un nuevo vaso precipitado con $30 \mathrm{ml}$ de agua destilada. Durante el periodo comprendido entre el día 3 y 30 las muestras se mantuvieron sin recambio de solución solo con agitación manual una vez al día. 
Resistencia Compresiva

La unidad de medida en que se obtuvo los resultados fue Mega Pascal (MPa). Se utilizó una máquina de ensayos universales (Lloyd, LR 100, UK). La máquina fue calibrada previa a la realización de la prueba utilizando 4 probetas de igual tamaño a las que se utilizarían en la medición. Los valores de resistencia compresiva fueron determinados para 40 probetas usando una máquina de ensayos universales con una velocidad de cruceta de $1 \mathrm{~mm} / \mathrm{min}$. Las muestras fueron cargadas hasta la fractura del material.

\section{Análisis de Datos}

Los datos se tabularán y analizarán con el programa estadístico SPSS 18 (IBM $®$ SPSS $®$ software). La hipótesis nula a probar será: la resistencia compresiva del sellante resinoso fluorado pre liberación de flúor (F1) es igual a la resistencia compresiva de sellante resinoso fluorado post liberación de flúor (F2). Las comparaciones entre los grupos F1 vs F2 y C1 vs C2 se analizará con el test t-Student. El nivel de significancia se establecerá en 0.05 .

\section{RESULTADOS}

\section{Resistencia Compresiva}

La resistencia compresiva de las muestras sin inmersión en agua fue de $337.20 \pm 62.50 \mathrm{MPa}$ para el sellante fluorado y $203.80 \pm 12.60$ MPa para el sellante no fluorado. Por otra parte, luego de la inmersión por 30 días en agua destilada, la resistencia compresiva fue $337.40 \pm 59.60$ $\mathrm{MPa}$ para el sellante fluorado y $213.40 \pm 46.90 \mathrm{MPa}$ para el sellante no fluorado. Estas diferencias entre grupos, tanto para las muestras sin inmersión como luego de estar expuestas al agua destilada no fueron significativas ( $p>0.05)$ como se observa en la Tabla 2.

Tabla 2. Resistencia compresiva.

\begin{tabular}{|l|lll|}
\hline \hline & \multicolumn{3}{|c|}{$\begin{array}{c}\text { Resistencia Compresiva } \\
\text { (MPa) (DE) }\end{array}$} \\
\hline & Seco & $\begin{array}{l}\text { Luego de 30 } \\
\text { días en agua }\end{array}$ & $p$ \\
\hline Con Flúor & $337.20 \pm 62.50$ & $337.40 \pm 59.60$ & 0.774 \\
\hline Control & $203.80 \pm 12.60$ & $213.40 \pm 46.90$ & 0.149 \\
\hline
\end{tabular}

\section{Liberación de Flúor}

La mayor liberación de flúor ocurrió a las 24 horas con una concentración de $1.90 \pm 0.50$ ppm, la cual decayó hasta llegar a 0.00 ppm el día 3. El detalle está disponible en la Tabla 3.

Tabla 3. Concentraciones diarias de flúor liberado desde sellante resinoso fluorado (grupo F2).

\begin{tabular}{|c|c|}
\hline Día & $\begin{array}{c}\text { Concentración de flúor } \\
(\boldsymbol{p p m}) \text { (DE) }\end{array}$ \\
\hline 1 & $1.90 \pm 0.50$ \\
\hline 2 & $0.20 \pm 0.00$ \\
\hline 3 & $0.0 \pm 0$ \\
\hline 30 & $0.0 \pm 0$ \\
\hline
\end{tabular}

\section{DISCUSIÓN}

La liberación de flúor desde el sellante resinoso fluorado no afectó su resistencia compresiva in vitro. Se encontraron valores de resistencia compresiva similares pre y post liberación de flúor luego de 30 días.

La metodología apropiada para medir resistencia compresiva y concentración de flúor liberado por el sellante resinoso fluorado corresponde a un estudio in vitro, sin embargo, esta metodología tiene algunas limitaciones inherentes.
Por una parte se utilizó agua destilada a $20^{\circ} \mathrm{C}$ en lugar de saliva artificial a $37^{\circ} \mathrm{C}$. Aun cuando la saliva artificial habría sido el medio ideal para hacer una comparación con la situación clínica de un sellante resinoso en boca, no es factible el uso de saliva artificial, rica en iones, ya que se altera la lectura del electrodo. Asimismo, la temperatura afecta también la lectura del electrodo. Sin embargo, el haber utilizado agua destilada pudo repercutir en una mayor liberación de flúor desde el sellante que la que se hubiese encontrado en saliva artificial ${ }^{(10)} \mathrm{o}$ en la saliva humana. De la misma forma, el mantener las muestras a $20^{\circ} \mathrm{C}$ pudo disminuir la velocidad de reacción de disolución de la sal fluorada, ampliando el periodo durante el que se realizó el proceso de liberación del que se hubiese encontrado a $37^{\circ} \mathrm{C}$. Sin embargo, para efectos del objetivo de nuestro estudio, esta situación es una ventaja, ya que permitiría evaluar los cambios en la resistencia compresiva del sellante resinoso fluorado provocados por una liberación de flúor.

Por otra parte, para realizar la medición de la concentración de flúor liberado por un sellante, se requería un volumen de a lo menos $50 \mathrm{ml}$ para que el electrodo pudiese estar completamente inmerso, lo que significó la utilización de 2 gramos de sellante resinoso fluorado por probeta. Esta cantidad difiere de las condiciones clínicas habituales, donde se utiliza usualmente una cantidad mucho menor de sellante por diente. Respecto a la medición de la concentración de flúor liberado desde el sellante resinoso fluorado, se evaluó la liberación de todas las caras en cada probeta, en concordancia al material y método utilizado en un estudio previo(6). Sin embargo, en condiciones clínicas, el sellante aplicado sobre fosas y fisuras, libera flúor solo desde la superficie oclusal, por lo que en condiciones clínicas liberaría menos fluoruros que en condiciones in vitro. Las limitaciones previamente señaladas implican que la concentración de flúor liberado en condiciones clínicas intraorales es menor a lo reportado en estudios in vitro y ante esto, es menos probable de que exista un efecto en la resistencia compresiva del sellante.

Nuestros resultados podrían explicarse debido a que la liberación de flúor desde el sellante resinoso fluorado causó una pérdida de masa insignificante para disminuir la resistencia del sellante ante una carga compresiva. Esta pérdida es mayor durante las primeras 24 horas de inmersión, y puede ser atribuida a iones fluoruro sujetos débilmente a la malla superficial del sellante resinoso, debido a que ésta es afectada por la inhibición de polimerización que causa la presencia de oxigeno ${ }^{(10)}$. Asimismo, la baja liberación de iones fluoruro observada luego de 48 horas de inmersión en agua, podría ser consecuencia de la baja concentración de flúor incorporada al sellante. Aun cuando por investigaciones previas indican que el sellante FluroShield contiene $2 \%$ de flúor ${ }^{(10)}$, los fabricantes no especifican la concentración de flúor en la resina ni la concentración de flúor que debería liberar.

Un estudio, con una metodología in vitro similar a la nuestra, encontró diferencias en la resistencia compresiva de sellantes resinosos fluorados luego de 28 días de liberación de flúor, específicamente para la marca Clinpro, 3M ESPE, USA ${ }^{(6)}$. Los valores de resistencia compresiva del sellante resinoso fluorado evaluado en el presente estudio (FluroShield, Dentsply, Brasil) son superiores a los reportados en el artículo de Kavaloglu Cildir ${ }^{(6)}$ tanto antes como después de la liberación de flúor. Las diferencias encontradas entre ambas investigaciones podrían explicarse por la distinta composición de ambos materiales fluorados, como por ejemplo, la fuente liberadora de flúor ya que en FluroShield, es fluoruro de sodio y en Clinpro es Tetrabutilamonio Tetrafluorborato. Sin embargo, nuestro estudio aporta información adicional al comparar los resultados contra un grupo control, lo que permite evaluar el efecto de la inmersión de la resina del sellante.

Considerando que el éxito de los sellantes oclusales se basa en su retención e integridad ${ }^{(4,5)}$ y no en su capacidad remineralizadora, una implicancia clínica de nuestros resultados es que la liberación de flúor desde un sellante resinoso fluorado no alteraría su resistencia compresiva, a diferencia de lo que ocurre con otros biomateriales de restauración que contienen flúor, como los composites, compómeros y cementos de vidrio ionómero, lo que le permitiría a los sellantes resinosos fluorados cumplir con su función para la prevención de caries.

\section{CONFLICTOS DE INTERÉS}

Los autores declaran no tener ningún vínculo con cualquiera de los productos mencionados en este estudio. 


\section{AGRADECIMIENTOS}

Los autores agradecen la colaboración de Eduardo Quiroz y Bernardo Jara del Instituto de Química. Facultad de Ciencias. Universidad Austral de Chile. Valdivia, Chile. A Andrés Siel y Víctor Vergara. De Ingeniería Mecánica. Universidad Tecnológica de Chile Inacap. Valdivia, Chile y a Danilo Bonadia Catani, Lívia Maria Andaló Tenuta y Jaime Aparecido Cury del Departamento de Ciências Fisiológicas, Área de Bioquímica.Faculdade de Odontologia de Piracicaba. Universida de Estadual de Campinas (UNICAMP), Piracicaba, SP, Brasil. Esta investigación fue parcialmente financiada por el Departamento de Asuntos Estudiantiles de la Universidad Austral de Chile y el Programa de Honor en Investigación de la Facultad de Medicina de la Universidad Austral de Chile. Valdivia, Chile. La presente investigación es parte del seminario de titulación de CV como parte de los requisitos para optar al Grado de Licenciado en Odontología. Escuela de Odontología, Facultad de Medicina, Universidad Austral de Chile.

\section{REFERENCIAS BIBLIOGRÁFICAS}

1. Kaste LM, Selwitz RH, Oldakowski RJ. Coronal caries in the primary and permanent dentition of children and adolescents 1-17 years of age: United States, 1988-1991. J Dent Res, 1996; 75: 631-641.

2. Ahovuo-Saloranta A, Hiiri A, Nordblad A, Mäkelä M, Worthington HV. Pit and fissure sealants for preventing dental decay in the permanent teeth of children and adolescents. Cochrane Database Syst Rev, 2008 Oct 8; 4: CD001830.

3. Gooch BF, Griffin SO, Gray SK, Kohn WG, Rozier RG, Siegal M, Fontana M, Brunson D, Carter N, Curtis DK, Donly KJ, Haering H, Hill LF, Hinson HP, Kumar J, Lampiris L, Mallatt M, Meyer DM, Miller WR, SanziSchaedel SM, Simonsen R, Truman BI, Zero DT. Centers for disease control and prevention. Preventing dental caries through school-based sealant programs: Updated recommendations and reviews of evidence. $J$ Am Dent Assoc, 2009 Nov; 140(11): 1356-1365.

4. Simonsen RJ. Glass ionomer as fissure sealant - a critical review. $J$ Public Health Dent, 1996; 56: 146-149.

5. Romcke RG, Lewis DW, Maze BD, Vickerson RA. Retention and maintenance of fissure sealants over 10 years. J Can Dent Assoc, 1990 Mar; 56(3): 235-237.
6. Kavaloglu Cildir S, Sandalli N. Compressive strength, surface roughness, fluoride release and recharge of four new fluoride-releasing fissure sealants. Dent Mater J, 2007 May; 26(3): 335-341.

7. Cury JA, Tenuta LMA. Enamel remineralization: Controlling the caries disease or treating early caries lesions? Braz Oral Res, 2009; 23(Spec Iss 1): 23-30.

8. Swartz ML, Phillips RW, Norman RD, Elliason S, Rhodes BF, Clark HE. Addition of fluoride to pit and fissure sealants-a feasibility study. J Dent Res, 1976 Sep-Oct; 55(5): 757-771.

9. Xu X, Burgess JO. Compressive strength, fluoride release and recharge of fluoride-releasing materials. Biomaterials, 2003 Jun; 24(14): 2451-2461.

10. Cury JA, Saad JC, Rodrigues Jr AL. Liberação de flúor do selante Na agua, saliva e soluções desmineralizante-remineralizante. RGO, 1993 Sep-Oct; 41(5): 273-275. 